\title{
On the limits of uniaxial magnetic anisotropy tuning by a ripple surface pattern
}

\author{
Miguel A. Arranz, ${ }^{1}$ Jose M. Colino, ${ }^{2, a)}$ and Francisco J. Palomares ${ }^{3}$ \\ ${ }^{1}$ Facultad de Ciencias Químicas, Universidad de Castilla-La Mancha, Avda. Camilo J. Cela 10, \\ 13071 Ciudad Real, Spain \\ ${ }^{2}$ Instituto de Nanociencia, Nanotecnología y Materiales Moleculares, Universidad de Castilla-La Mancha, \\ Campus de la Fábrica de Armas, 45071 Toledo, Spain \\ ${ }^{3}$ Instituto de Ciencia de Materiales de Madrid, Consejo Superior de Investigaciones Científicas, \\ c/ Sor Juana Inés de la Cruz 3, 28049 Madrid, Spain
}

(Received 4 April 2014; accepted 30 April 2014; published online 13 May 2014)

\begin{abstract}
Ion beam patterning of a nanoscale ripple surface has emerged as a versatile method of imprinting uniaxial magnetic anisotropy (UMA) on a desired in-plane direction in magnetic films. In the case of ripple patterned thick films, dipolar interactions around the top and/or bottom interfaces are generally assumed to drive this effect following Schlömann's calculations for demagnetizing fields of an ideally sinusoidal surface [E. Schlömann, J. Appl. Phys. 41, 1617 (1970)]. We have explored the validity of his predictions and the limits of ion beam sputtering to induce UMA in a ferromagnetic system where other relevant sources of magnetic anisotropy are neglected: ripple films not displaying any evidence of volume uniaxial anisotropy and where magnetocrystalline contributions average out in a fine grain polycrystal structure. To this purpose, the surface of $100 \mathrm{~nm}$ cobalt films grown on flat substrates has been irradiated at fixed ion energy, fixed ion fluency but different ion densities to make the ripple pattern at the top surface with wavelength $\Lambda$ and selected, large amplitudes $(\omega)$ up to $20 \mathrm{~nm}$ so that stray dipolar fields are enhanced, while the residual film thickness $t=35-50 \mathrm{~nm}$ is sufficiently large to preserve the continuous morphology in most cases. The film-substrate interface has been studied with X-ray photoemission spectroscopy depth profiles and is found that there is a graded silicon-rich cobalt silicide, presumably formed during the film growth. This graded interface is of uncertain small thickness but the range of compositions clearly makes it a magnetically dead layer. On the other hand, the ripple surface rules both the magnetic coercivity and the uniaxial anisotropy as these are found to correlate with the pattern dimensions. Remarkably, the saturation fields in the hard axis of uniaxial continuous films are measured up to values as high as $0.80 \mathrm{kG}$ and obey a linear dependence on the parameter $\omega^{2} / \Lambda / t$ in quantitative agreement with Schlömann's prediction for a surface anisotropy entirely ruled by dipolar interaction. The limits of UMA tuning by a ripple pattern are discussed in terms of the surface local angle with respect to the mean surface and of the onset of ripple detachment. (C) 2014 AIP Publishing LLC. [http://dx.doi.org/10.1063/1.4876232]
\end{abstract}

\section{INTRODUCTION}

Ion beam sputtering (IBS) of solid matter has demonstrated to be a handy method in many labs to induce selforganized nanoscale surface patterns. At a broad range of beam parameters, a ripple pattern forms in the irradiated surface. For decades, experiments and theories have unveiled that ripple formation originates from the competition of two counteracting processes: first, the curvature dependent ion induced surface instability that amplifies the roughness, and second, the diffusion, which tends to flatten the surface. Detailed explanations of the overall ripple formation process have been published elsewhere. ${ }^{1,2}$ The versatility of this technique for surface patterning originates from only several irradiation parameters, like ion energy, angle of incidence, ion flux, ion fluency, and so forth. Also the initial surface morphology is known to profoundly determine the

\footnotetext{
a) Author to whom correspondence should be addressed. Electronic mail: josemiguel.colino@uclm.es.
}

subsequent ripple pattern evolution. Some methods control the surface morphology of the virgin films by means of different deposition procedures, which result in a variety of mean grain sizes that affects the lateral periodicity of the ripple pattern, ${ }^{3}$ alternatively other methods gain control of the ripple pattern amplitude by means of a prior chemical etching of the non-irradiated wafer $^{4}$ or making use of kinetic roughening in PVD films. ${ }^{5}$

A number of studies on ripple formation have been carried out on technologically important, polycrystalline metal films. Ion beam techniques have already been tested to improve magnetic read-write properties and thermal stability of $\mathrm{CoCrPtB}$ active layers in longitudinal recording media. ${ }^{6}$ On the fundamental viewpoint, the control of magnetic anisotropy in low dimensional system has emerged as an important topic in the nanomagnetism research. Single polycrystalline films of permalloy, iron, or cobalt deposited on patterned surfaces exhibit substantial modifications of their magnetic properties, ${ }^{7-11}$ most noticeably the appearance of uniaxial magnetic anisotropy (UMA). Such induced 
anisotropy has also been used to control the interlayer exchange coupling in $\mathrm{Fe} / \mathrm{Cr} / \mathrm{Fe}$ trilayers ${ }^{8}$ or exchange biased multilayers. ${ }^{9}$ In the literature, we basically find two methods of imprinting UMA in magnetic layers by IBS techniques: on one hand, ion erosion is applied to a substrate, usually a silicon wafer, to make ripples in its surface, i.e., to prepattern the surface onto which the magnetic film can be subsequently grown; ${ }^{12-15}$ on the other hand, the magnetic film is first deposited on a polished substrate, later an ion beam erodes directly the film surface to induce a ripple pattern. ${ }^{16,17}$ Other authors have pursued different strategies, for instance, Umlor has found UMA to be related to oblique deposition of a non-magnetic buffer layer; ${ }^{18}$ similarly Yakovlev et al. have made use of the intrinsic groove morphology of the $\mathrm{CaF}_{2} / \mathrm{Si}\left(\begin{array}{lll}1 & 0 & 0\end{array}\right)$ interface to form a ripple pattern on the cobalt films grown atop. ${ }^{19}$

Pioneering research on the UMA induced in ripple films performed at the Göttingen and Dresden groups has focused mainly on the ripple wavelength and thickness dependence of the anisotropy energy density. ${ }^{7,8,12,16,17}$ Following their articles, we have learned that the origin of UMA imprinted in polycrystalline films can be different from that in epitaxial films and was interpreted with magnetostatic contributions of the overall magnetic anisotropy, e.g., shape anisotropy and dipolar interactions. In either single-crystal or polycrystalline films, a relevant feature of the film morphology is whether the roughness profiles of the top and bottom interfaces are correlated with each other. Films deposited on prepatterned substrates usually exhibit correlated interfaces, and thus the magnetization profile substantially depends upon the film thickness; Vaz et al. distinguished two thickness regimes in that scenario: ${ }^{20}$ (i) A thin film regime, where the magnetization follows the roughness profile, thus the magnetostatic energy is reduced due to "orange-peel" effects. (ii) A thick film regime, where the linear increase of exchange and anisotropy energy brings the system to the uniform magnetic state, e.g., dipolar charges act as a surface anisotropy contribution that is superimposed on the volume contributions. However, detailed studies have shown a more complicated magnetization profile underneath the ripple surface; Liedke et al. performed micromagnetic simulations ${ }^{12}$ in films with coherent interfaces indicating a thickness dependent magnetization tilt in the thick film regime. Starting at the surface, the magnetization tilt decreases with increasing depth until it reaches its smallest tilt in the middle of the layer. In the case of films with only one rough interface (top surface), such as a ripple pattern surface resulting from direct ion erosion, we expect a smaller effect of dipolar interactions and a more uniform magnetization profile. This kind of films resembles closely the ideal scenario of a single, rough interface for which Schlömann calculated the demagnetizing fields ${ }^{21}$ arising solely from the surface poles. However, in such films, there is no published experimental work or micromagnetic simulation, which we are aware of, studying the magnetization tilt at and below the top surface layers.

In this paper, we explore the UMA of films with a ripple pattern at the top surface only, films as thick as possible to minimize a possible volume contribution (usually associated with lattice strain and expected to fade away with increasing thickness), films where the magnetocrystalline energy averages out because of the fine grain polycrystal structure, thus where the surface magnetostatic contribution to the anisotropy field can straightforwardly be compared to a prediction. According to Schlömann's calculations, ${ }^{21}$ the demagnetizing field of a periodic ripple surface depends on ripple wavelength $\Lambda$, film thickness $t$, and ripple amplitude $\omega$ as $\omega^{2} / \Lambda /$ t. Since the amplitude enters the formula as squared, our strategy has been to consider it as the leading variable upon which the law is to be checked, while the thickness and ripple wavelength are as fixed as possible.

\section{EXPERIMENTAL METHODS}

Nano-undulated surface patterns have been fabricated on cobalt films in a double-chamber vacuum system. Prior to insertion into the deposition chamber, the substrates were cleaned in an ultrasonic acetone bath to eliminate organic contaminants and rinsed in pure water. Base pressure before deposition was $4 \times 10^{-8}$ millibars. Polycrystalline cobalt films were grown on unheated $\mathrm{Si}\left(\begin{array}{lll}1 & 0 & 0\end{array}\right), \mathrm{MgO}\left(\begin{array}{lll}1 & 0 & 0\end{array}\right)$, or glass (Corning) substrates by dc sputtering from a $1.5 \mathrm{in}$. diameter magnetron source in the argon $8 \times 10^{-3}$ millibars pressure range. The substrate is located at the center above and parallel to the sputter target to rid the growing film of any features related with geometrical effects. Most part of the analysis reported in this paper is relative to films on $\mathrm{Si}(1$ 00 ), thus particular interest deserves the study of the $\mathrm{Co} / \mathrm{Si}$ interface; nonetheless, the use of a thermally more stable substrate, such as $\mathrm{MgO}\left(\begin{array}{lll}1 & 0 & 0\end{array}\right)$ or glass, is complementary. The thickness of as-grown films is determined at $\sim 100 \mathrm{~nm}$ by ex situ X-ray reflectivity. This initial thickness was chosen to make polycrystalline films without a texture and with a rough virgin surface $(\mathrm{rms} \sim 2 \mathrm{~nm})$, the latter allowing to sculpt a deep surface pattern with a subsequent ion erosion. ${ }^{5}$ Just after deposition, the film is transferred in vacuum to a separate chamber for ion beam irradiation. This process chamber is equipped with a wide beam $(\varnothing 3 \mathrm{~cm})$ Kauffman type dc ion source. Irradiation takes a beam of $1.2 \mathrm{keV} \mathrm{Ar}^{+}$ ions at an incidence angle of $80^{\circ}$ with respect to the surface normal of the target film. At a large enough fluency, the process makes a surface ripple pattern with a wave vector perpendicular to the ion beam direction. ${ }^{5,22}$ Within the irradiation stage, the ion energy was fixed while the ion flux was varied from run to run by tuning the beam current in the power source. By doing this, we intended to make ripple patterns of greater depth/amplitude, i.e., large surface roughness. Previous evidence of such effect is referred to in a paper by Chason et al.; ${ }^{23}$ experiments with an ion flux up to $3 \mu \mathrm{A} / \mathrm{cm}^{2}$ and fluencies up to $6 \times 10^{16} \mathrm{~cm}^{-2}$ have proved that the surface roughness increases as the square of the flux. In turn, our ion source works in a range of large ion density higher than about $100 \mu \mathrm{A} / \mathrm{cm}^{2}$ (equivalent to a projectile flux $0.63 \times 10^{14} \mathrm{ions} / \mathrm{s} / \mathrm{cm}^{2}$ ). In order to probe the effect of different ion fluxes, the actual etch rate is determined by measuring the film thickness before and after ion irradiation by either X-ray reflectivity on a separate set of eroded films or by atomic force microscopy (AFM) line profiling across substrate-to-surface steps made with optical lithography. 
On the other hand, since we intended to make ripple films of about the same residual thickness, the irradiation time has been adjusted in each run to keep the ion fluency fixed at around $4 \times 10^{18} \mathrm{~cm}^{-2}$. Finally, after the ion erosion, every sample is in-situ capped with a $2 \mathrm{~nm} \mathrm{AlO}_{\mathrm{X}}$ layer.

$\mathrm{X}$-ray diffraction (XRD) is measured in a conventional $\mathrm{Cu} \mathrm{K} \alpha$ radiation diffractometer including a curved graphite monochromator in Bragg-Bentano's geometry at high angles of incidence $(\theta) . \theta / 2 \theta$ scans show the three lines characteristic of polycrystalline hcp cobalt in the as-grown film, also in the patterned films, without any evidence of crystallographic texture. ${ }^{5}$ Line width analysis of these lines indicates a crystalline coherence of $8 \pm 1 \mathrm{~nm}$, thus proving the fine grain polycrystal microstructure. The surface topography was investigated $e x$ situ by AFM operating in tapping mode. All measurements were conducted in air at room temperature using commercial silicon cantilevers. AFM scan size is typically $2 \times 2 \mu \mathrm{m}^{2}$. At this image size, the calculated surface roughness has reached a saturation regime because the sampling window becomes comparable with or greater than the pattern feature size.

X-ray photoelectron spectroscopy (XPS) depth profiling experiments allowed us to get information on the chemical composition along the sample thickness. XPS spectra were acquired in an ultrahigh vacuum (UHV) chamber with a base pressure of $10^{-9}$ millibar using a hemispherical electron energy analyzer (SPECS Phoibos 150 spectrometer) and a monochromatic AlK $\alpha(1486.74 \mathrm{eV}) \mathrm{X}$-ray source. XPS spectra were recorded at normal emission take-off angle, using an energy step of $0.05 \mathrm{eV}$ and a pass-energy of $20 \mathrm{eV}$ for atomic quantification purposes. High resolution data taken with energy step and pass energy of $0.025 \mathrm{eV}$ and $7 \mathrm{eV}$, which provide an overall instrumental peak broadening of $0.4 \mathrm{eV},{ }^{24}$ were used for lineshape and deconvolution analysis. Carbon and hydroxyl $(\mathrm{OH})$ species were also detected as contaminants on the surface of the samples, and the signal from adventitious carbon at $284.6 \mathrm{eV}$ was used for energy calibration. The overall surface composition was determined from survey spectra and regions of interest (Co2p, O1s, C1s, and $\mathrm{Si} 2 \mathrm{p}$ ). The continuous erosion of the sample produced by the sequence of $\mathrm{Ar}^{+}$ion bombardment $(1 \mathrm{KeV}$, $1.5 \mu \mathrm{A} / \mathrm{cm}^{2}, 0.15 \mathrm{~nm} / \mathrm{min}$ etch rate) cycles interleaved with XPS measurements provides the means of analyzing the composition at different depth steps from the film surface to the Si substrate. The integral peak areas after background subtraction and normalization using sensitivity factors provided by electron energy analyzer manufacturer were used to calculate the atomic concentration of each element. Data processing was performed using CasaXPS software (Casa Software Ltd., Cheshire, UK).

The magneto-optical Kerr effect (MOKE) was measured with our experimental setup for vectorial magnetometry. ${ }^{25,26}$ The He-Ne laser beam $(633 \mathrm{~nm}, 1 \mathrm{~mW})$ was focused on the sample at an incidence angle of $45^{\circ}$ relative to its normal axis. A high grade Glann-Thompson polarizer was used to align the radiation polarization plane parallel to the incident plane, coinciding with the external magnetic field, $\mathrm{H}$ (longitudinal MOKE geometry). As is well known, this configuration allows one to simultaneously investigate both in plane magnetization components, parallel and perpendicular to $\mathrm{H}$, respectively. In this work, for measuring parallel magnetization $\mathrm{M}_{\mathrm{PAR}}$, the external $\mathrm{H}$ was applied in the range of \pm 1.50 $\mathrm{kG}$ at a sweep rate of $15 \mathrm{~Hz}$. The reflected beam from the sample was recorded with ultrafast photodiodes and an amplifier circuit. The angular dependence of $\mathrm{M}_{\mathrm{PAR}}$ was studied by rotating the sample holder around its normal axis, $\theta$, being equivalent to rotate the external field in the sample plane. Finally, statistical noise in $\mathrm{M}_{\mathrm{PAR}}$ measurement was further reduced by averaging many measurements at fixed conditions.

\section{RESULTS AND DISCUSSION}

\section{A. Film surface morphology}

The left column of Figure 1 shows representative AFM images of films eroded at a fixed ion fluency $\varphi \approx 4$ $\times 10^{18} \mathrm{~cm}^{-2}$ with different ion fluxes/etch rates, increasing from the top image $(0.013 \mathrm{~nm} / \mathrm{s})$ to the bottom image $(0.040 \mathrm{~nm} / \mathrm{s})$. All samples have developed a ripple pattern along the projected direction of the ion beam (see arrows), as generally observed in the erosive regime. The average ripple length is around $0.5 \mu \mathrm{m}$ or greater. $\mathrm{K}$-space 2D-FFT maps (insets) show elongated spots indicating similar pattern coherence irrespective of the ion flux and a predominant roughening in the direction perpendicular to the projected beam. In order to analyze the surface features of every image, we have statistically performed the ridge-to-valley excursions in a set of line profiles measured in the direction of the pattern wavevector, i.e., perpendicular to the ripple crests. The ripple wavelength histograms for the images on the left column are displayed in the center column of Figure 1. A wavelength distribution is found with the mean lying in the range of $60-75 \mathrm{~nm}$; these values vary without any clear dependence on the ion flux. We think that those small changes of the mean ripple spacing are related to uncontrollable small variations $\left(<2^{\circ}\right)$ of angle of incidence from one run to the next, since in previous experiments at around an angle of incidence of $80^{\circ}$, such small variations have proved a profound effect on the ripple wavelength which was explained in accord with the Bradley-Harper theory. ${ }^{27}$ More significant is the ripple amplitude histograms of the same images shown in the right column of Figure 1. The trend is clear: the mean ripple amplitude increases with a higher ion flux. Also the amplitude distribution becomes broader with increasing ion flux, but the greatest amplitudes do not reach the value corresponding to the average thickness, hence these films can be considered as continuous.

Figure 2(a) summarizes the effect of the increasing ion flux/etch rate on the ripple pattern amplitude at fixed ion fluency. The ripple amplitude of films grown on either $\mathrm{Si}\left(\begin{array}{lll}1 & 0 & 0\end{array}\right)$ or glass increases with the etch rate following the same qualitative dependence as reported by Chason et al. ${ }^{23}$ The same trend is observed with the rms roughness, although at smaller values because it accounts for both $\mathrm{X}$ and $\mathrm{Y}$ directions. Therefore, from these results, the ion flux seems to be responsible for tuning the ripple amplitude.

\section{B. Film-substrate interface}

There has been a considerable interest in the study of formation of a magnetic dead layer at the interface of 
Ripple Wavelength (nm)
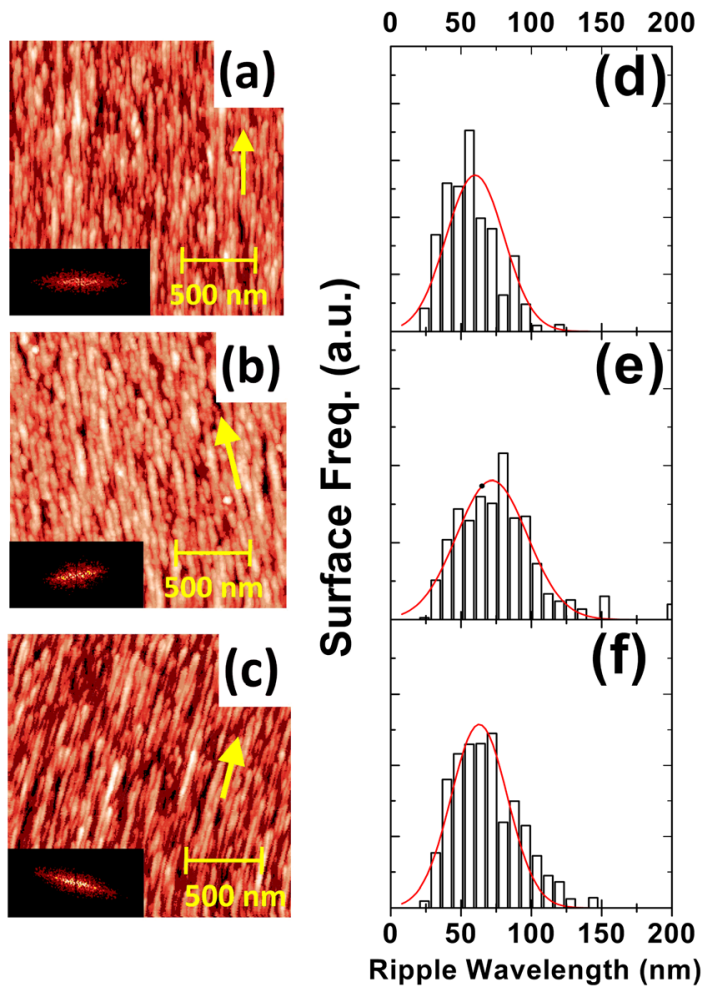

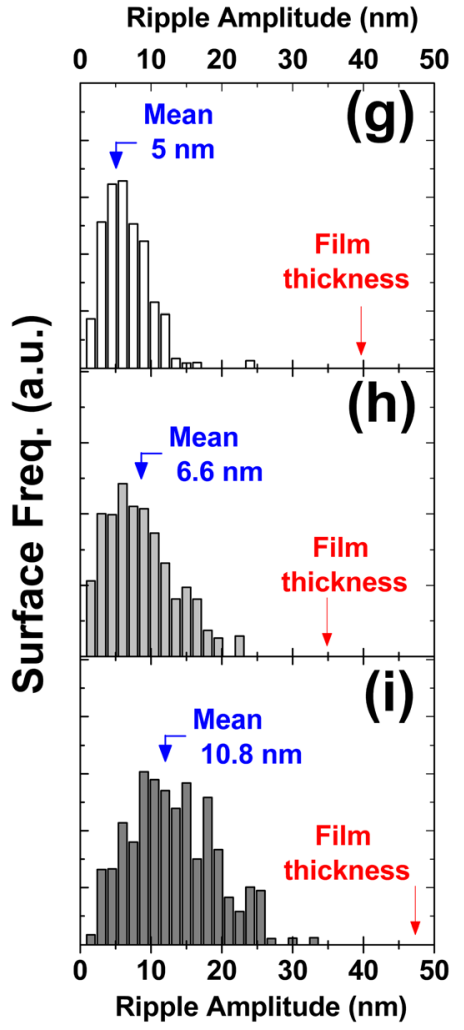

FIG. 1. Left column: Atomic force microscopy images of $2 \times 2 \mu \mathrm{m}^{2}$ surface of cobalt films eroded at an ion fluency $\varphi \approx 4 \times 10^{18} \mathrm{~cm}^{-2}$ with different ion fluxes/etch rates; (a) $0.013 \mathrm{~nm} / \mathrm{s}$, (b) $0.025 \mathrm{~nm} / \mathrm{s}$, and (c) $0.040 \mathrm{~nm} / \mathrm{s}$. K-space 2D Fourier (2D-FFT) maps are included as insets for a range of $100 \mu \mathrm{m}^{-1} \times 50 \mu \mathrm{m}^{-1}$. Center column: Histograms of surface occupancy versus ripple spacing for the same films as previously, with plots (d), (e), and (f) corresponding to erosion rates 0.013 , 0.025 , and $0.040 \mathrm{~nm} / \mathrm{s}$, respectively. Right column: Histograms of surface occupancy versus ripple amplitude, plots (g)-(i) corresponding to the same erosion rates as that of the other columns. magnetic and non-magnetic layers, as they can significantly influence the overall magnetic properties of the film and multilayered thin film structures. ${ }^{15,28}$ Either because of some intermixing or some structural modifications at the interface,

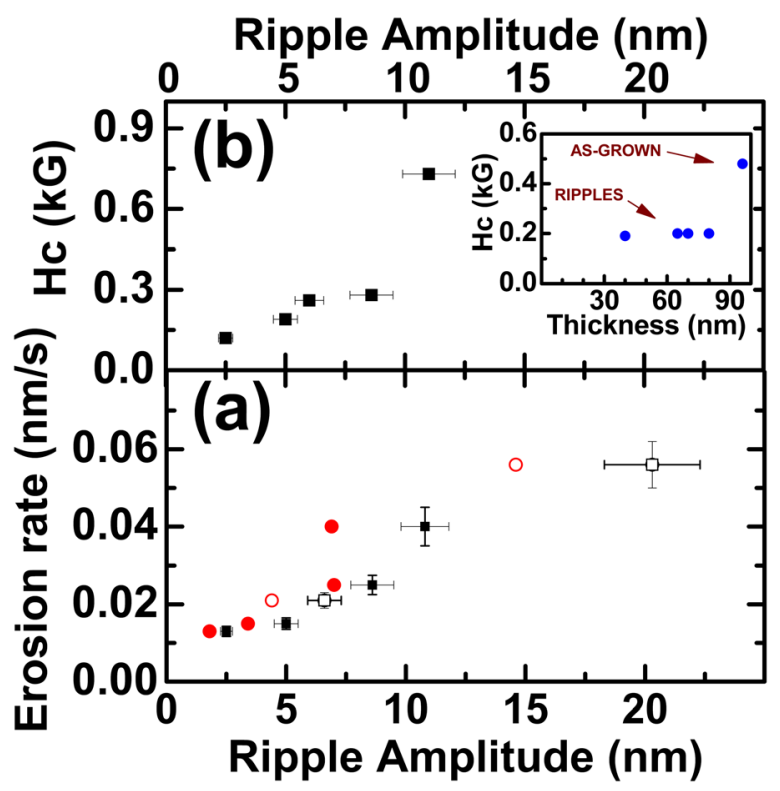

FIG. 2. (a) Ion erosion rate as a function of the ripple pattern amplitude for films grown on either silicon (solid symbols) or glass (open symbols). Circle data correspond to rms (image) roughness. Squares are the mean ripple amplitude statistically determined in the pattern wavevector direction. (b) Coercive field as a function of the ripple amplitude for pattern films on silicon at different etch rates. The inset shows the coercive field as a function of residual thickness for a set of films eroded at fixed etch rate $(0.013 \mathrm{~nm} / \mathrm{s})$ and varying ion fluency; once some layer is etched out, the surface pattern developed with an amplitude $5 \mathrm{~nm}$ and the coercive fields are irrespective of the residual thickness. a distinct, magnetically active layer or a dead layer can form at the interface. In case of ion irradiated films, the ion beam itself causes sample heating that could enhance intermixing and other interface modifications. Particularly, in the cobaltsilicon system, previous works have reported on the relevance of alloying on the magnetic properties: First, single $\mathrm{Co}_{1-\mathrm{X}}-\mathrm{Si}_{\mathrm{X}}$ alloy films are known to retain the magnetic properties with the saturation magnetization vanishing only at $\mathrm{X}$ higher than $0.25,{ }^{29}$ since silicon as an additive is supposed to change Co alloys as a metalloid element. Other authors have established the maximum silicon content at $0.4^{30}$ or $0.35,^{31}$ also with the Si-richer side being non-magnetic. Second, alloy films in the $0.25<\mathrm{X}<0.35$ composition range are amorphous ferromagnets that can exhibit a well-defined UMA, ${ }^{32}$ the orientation of the magnetic easy axis being correlated with the expected anisotropic spatial distribution of $\mathrm{Si}$ concentration (which presumably arose from the film co-deposition geometry). All these evidences have raised our concern on the cobalt-silicon interface, leading us to perform a composition analysis by the XPS technique. According to the morphology results by microscopy experiments Figures 1(g)-1(i), the ripple film patterned at low ion current density is most adequate for depth profiling because the surface height distribution is not as broad as others.

A set of high-resolution Co2 $\mathrm{p}_{3 / 2}$ and Si2p XPS spectra corresponding to stages across the film-substrate interface are selected and displayed in Figure 3 to show the trend in the quantification values as a function of etch-time in the depth profile experiment. The upper Co2 $\mathrm{p}_{3 / 2}$ spectrum (label A) corresponds to characteristic emission from pure metallic Co; its evolution and comparison with signals from next stages readily reveals the line-shape broadening of the 

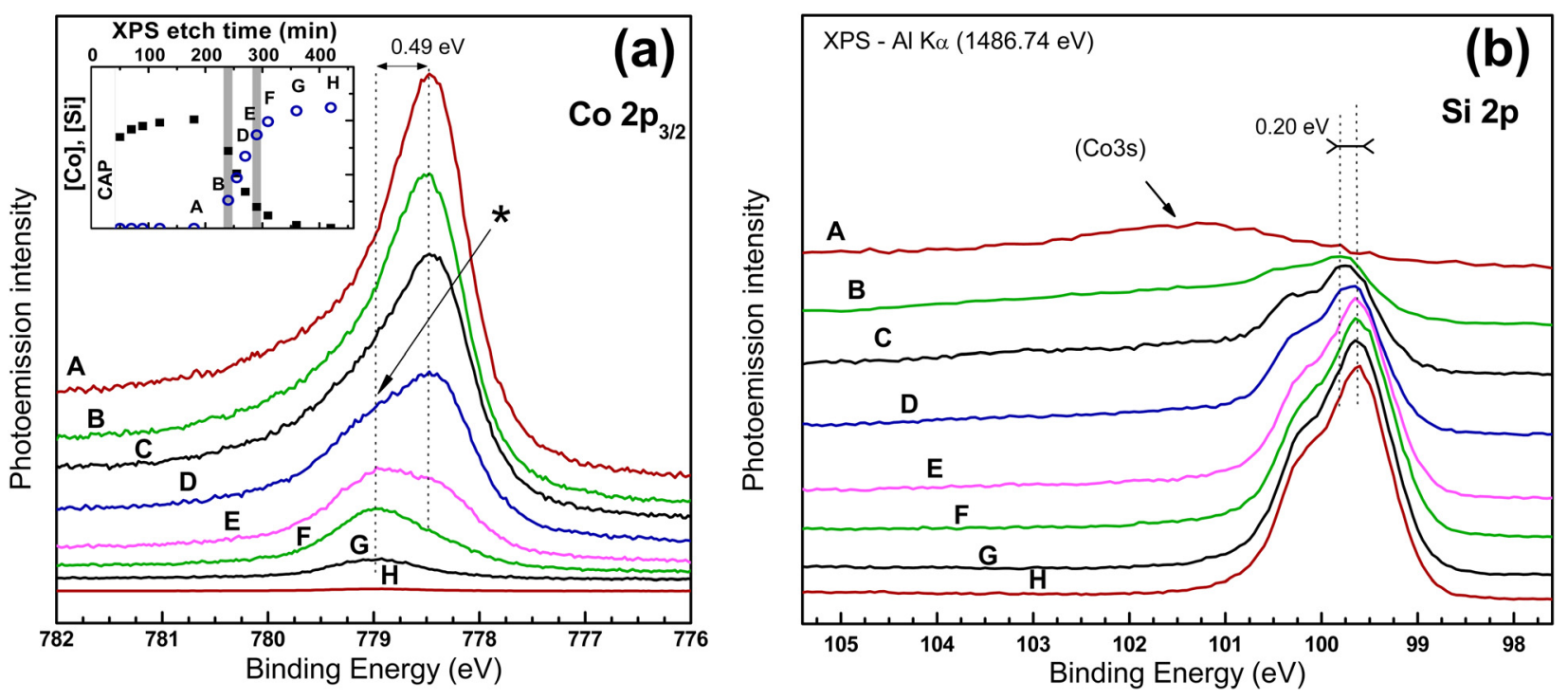

FIG. 3. High resolution XPS spectra of the evolution of (a) Co2 $\mathrm{p}_{3 / 2}$ and (b) Si2p core-levels for the ripple film patterned with low ion density ( $0.013 \mathrm{~nm} / \mathrm{s}$ ). Residual film thickness is $40 \mathrm{~nm}$. From bottom to top, the spectra displayed correspond to several depth profile stages (labels A-H) with increasing etch time across the film-substrate interface. The inset in (a) plots the depth profile of photoemission intensities—solid and empty symbols corresponding to cobalt and silicon, respectively, where the labels denote the profile stages referred to above.

spectra, which might be a clear indication of the chemical reaction of Co taking place with the substrate. Detailed line-shape analysis, upon normalization and overlapping of the pure Co and the second from top XPS spectra, from which $\mathrm{Si}$ emission is first observed, there appears a clear asymmetry on the high binding energy side. This effect is more prominent in consecutive spectra at given depth as profile develops. This fact is illustrated with the asterisk in the figure, which resembles the appearance of a chemically shifted component at $+0.50 \mathrm{eV}$ associated to silicide formation. This observation is also confirmed by the simultaneous existence of a chemical shift in its counterpart Si2p core level, which reaches a very small, but detectable, value $(+0.20 \mathrm{eV})$ due to the high resolution data obtained in the XPS experiments with monochromatic radiation. Both results are in agreement to the previous values reported in the literature ${ }^{33-35}$ and suggest the formation of silicide at the interface. In addition, to unequivocally determine the formation of silicide at the interface, spectra do not only reveal line-shape changes but also they are to be decomposed into chemically shifted components associated to the Co-silicide phases. Therefore, $\mathrm{Co} 2 \mathrm{p}_{3 / 2}$ and Si2p spectra characteristic of regions highlighted in the inset of Figure 3 are normalized to the same height to emphasize line shape changes and get information on subtle differences and deconvoluted (Figure 4). Both top Co2 $\mathrm{p}_{3 / 2}$ and $\mathrm{Si} 2 \mathrm{p}$ correspond to the first stage in which $\mathrm{Si}$ emission is detected, and silicide reaction is present. On the one hand, Co2 $\mathrm{p}_{3 / 2}$ is decomposed into Co-metal and Co-silicide components, after background subtraction. On the other hand, Si2p is decomposed into the $2 p_{3 / 2,1 / 2}$ doublet from silicide, Co3s and Si-oxide components. One point to notice about is the identification of the full amount of $\mathrm{Si}$ is in the form of silicide. This first stage of silicide formation resembles to the Co-richest one with composition $\mathrm{Co}_{1-\mathrm{X}}-\mathrm{Si}_{\mathrm{X}}$ of $\mathrm{X} \approx 0.7$, which corresponds to a non-ferromagnetic silicide phase. The subtle Si-oxide component present comes from the experimental conditions used for the growth of the Co films before its nanopatterning by ion irradiation since Co film is deposited on a clean Si substrate with a very thin native oxide layer. There also exists a component denoted by Co3s emission due to the fact that there is at this given depth, where silicide reaction starts taking place, a remaining non-reacted Co metal on top. Similarly, at slightly higher depth, represented by bottom spectra in Figure 4, both signals progress simultaneously and show a decrease/increase of Co-metal/Co-silicide signals with composition value of $\mathrm{X}=0.8$, and also the emission of $\mathrm{Si} 2 \mathrm{p}$ doublet coming from the $\mathrm{Si}$ substrate besides a small contribution from silicide into the Si2p signal. Next, consecutive deeper steps of analysis provide a continuous decrease in the amount Co in the silicide, following the evolution from pure Co layer through the Si-rich Co-silicide to the Si substrate. This characterization evidences the formation of a very narrow Co-silicide interface with gradually averaged content $\mathrm{X}>0.7$ with concentration well above the percentage to be considered as a magnetically dead layer. ${ }^{31}$ XPS depth profiles performed on virgin cobalt films (not shown) have also demonstrated the formation of a graded interface such as that of ripple films. Because of this, we think that the Co-silicide forms at the film growth and the ion irradiation is not modifying the film-substrate interface.

\section{Magnetostatic properties: Coercivity and uniaxial magnetic anisotropy}

The interplay of an isotropic surface roughness and coercive force has been studied from the experimental and theoretical approaches ${ }^{36-39}$ but, to our knowledge, there is no published work studying directly the relation between the coercive field and an anisotropic roughness profile such as a ripple surface of a magnetic film. The coercive force $\mathrm{Hc}$ of the ripple films has been measured along the ripple crest direction: Hc data are plotted in Figure 2(b) versus the ripple 


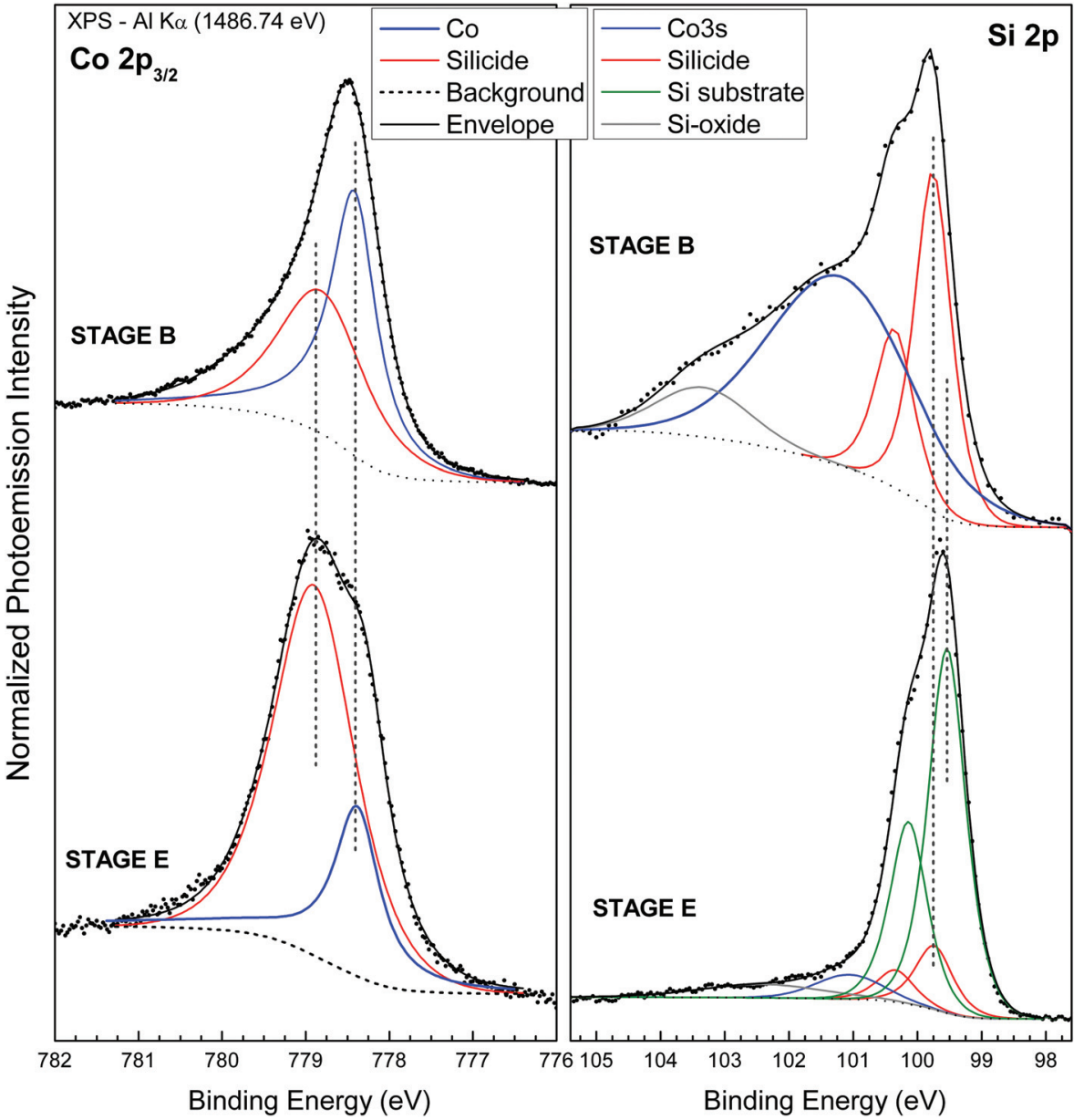

FIG. 4. High resolution XPS spectra of the evolution Co2 $\mathrm{p}_{3 / 2}$ and $\mathrm{Si} 2 \mathrm{p}$ core levels with spectral decomposition (see text for description of components). Upper and lower curves correspond to profile stages B and E, respectively, referred to in the inset of Figure 3 with labels and vertical grey stripes. amplitude. As we shall discuss later, this direction can be considered the easy axis. Zhao et al. ${ }^{36}$ have calculated Hc for both Bloch and Néel types of domain wall as a function of (isotropic) roughness amplitude or average surface slope and quantitatively compared it with experimental studies. In case of domain wall movement, the coercive field was predicted to increase linearly with roughness, whereas the rotational coercivity is either independent or decreases with increasing roughness, depending upon the wall type. ${ }^{36}$ Although their predictions apply to self-affine rough surfaces and were not extended to the case of anisotropic roughness, we note in Figure 2(b) the same dependence of Hc on the roughness amplitude as in the isotropic roughness scenario. This suggests that the coercive force for fields applied along the ripples is governed by wall movement without a major effect of the pattern. The inset of Figure 2(b) shows Hc data for a separate series of films eroded at a fixed ion flux, therefore, with fixed ripple amplitude, but with different fluencies (decreasing residual thickness) starting from 0 ions $/ \mathrm{cm}^{2}$ (as-grown film). Clearly, the Hc data for the virgin and ripple surfaces are very different, although, remarkably the latter do not depend on the residual thickness. This finding is consistent with the Hc dependence sketched above: the roughness amplitude is the leading factor on the easy axis coercivity.

Figure 5(a) displays the in-plane normalized magnetization MOKE cycles for the same three films representative of low, medium, and high ion flux patterning as those used in the previous morphology analysis. If the field is scanned along the direction perpendicular to the crests (hard-axis), the ripple pattern increases drastically the demagnetizing factor and the uniaxial magnetic anisotropy sets in; the loop includes a negligible coercive force and saturates at a field Hs (anisotropy field). We have determined the Hs value by the field corresponding to the maxima of the field derivatives of the relative magnetization $\mathrm{M} / \mathrm{M}_{\mathrm{S}}$ in the hard-axis loop. On the other hand, if the field is applied parallel to the crests (easy-axis), the experiments have shown loops of high squareness with coercive fields Hc not quite smaller than Hs (Hc/Hs $\sim 0.60-0.75)$. Though the squareness is only perfect for the lowest ion flux sample, in between the easy and the hard directions the relative remanence evolves as a function of in-plane field angle confirming the existence of a well-defined uniaxial magnetic anisotropy in all cases (Figure 5(b)).

Some authors consider that $\mathrm{H}_{\mathrm{S}}$ can be separated into a volume contribution $\mathrm{H}_{\mathrm{S}} \mathrm{V}$ and the surface contribution $\mathrm{H}_{\mathrm{S}}{ }^{\mathrm{S}}$ according to $\mathrm{H}_{\mathrm{S}}=\mathrm{H}_{\mathrm{S}}{ }^{\mathrm{V}}+\mathrm{H}_{\mathrm{S}} \mathrm{S} / \mathrm{t}$. Evidence of the volume contribution has been reported by Sarathlal et al., ${ }^{15}$ for example, with a weak UMA in as-grown Co films on polished $\mathrm{Si}$ wafers if the thickness is higher than $10 \mathrm{~nm}$, which was attributed to the generation of some long range internal stresses during the film deposition. A volume contribution could also be related to ripple dislocations where a broken symmetry would favour alignment of magnetic moments parallel to the ripple crests. For ripple films on pre-pattern 
Field (kG)
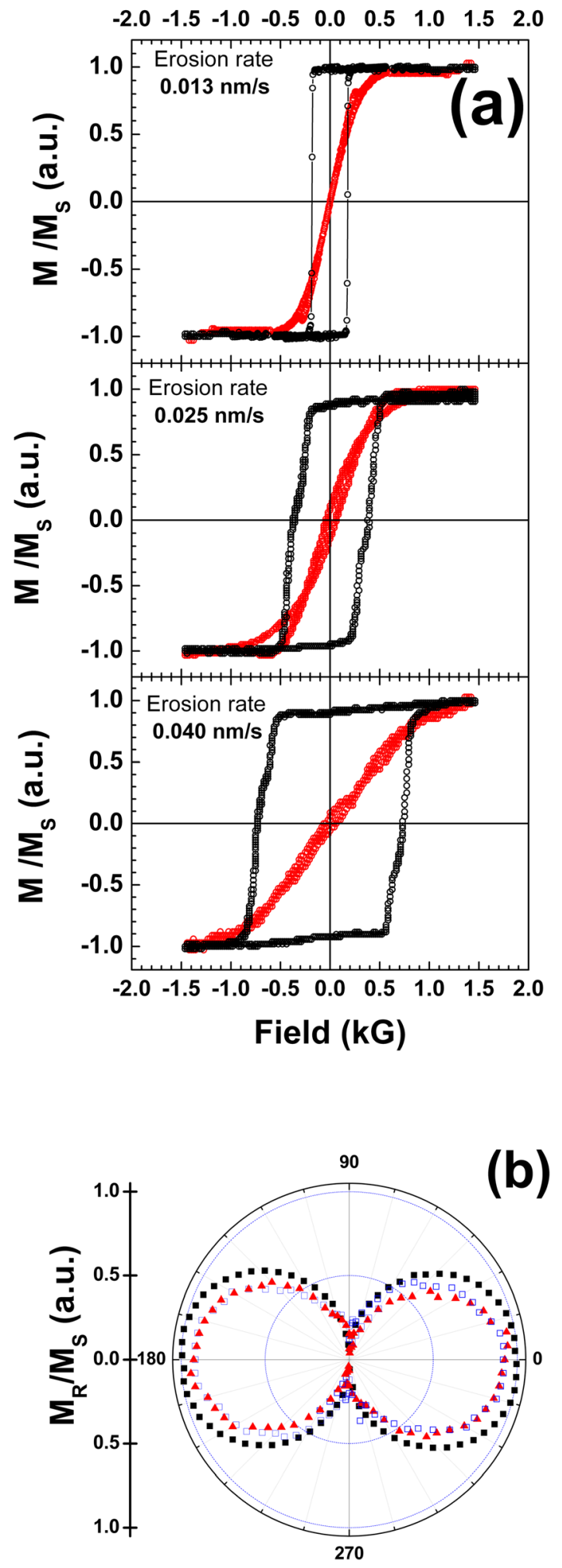

FIG. 5. (a) Normalized magnetization reversal $\mathrm{M} / \mathrm{M}_{\mathrm{S}}$ investigated by MOKE for two different in-plane directions: Black symbols correspond to field applied along the ripple pattern direction (easy axis) and red symbols correspond to field perpendicular to the ripple direction (hard axis). From top to bottom, the MOKE cycles were measured in the same films as those of Figure 1 for different etch rates. (b) Polar plot of the relative remanence $M_{R} / M_{S}$ for the same three films: Solid squares are data for the film eroded at the lowest rate $(0.013 \mathrm{~nm} / \mathrm{s})$, open squares and solid triangles correspond to films eroded at 0.025 and $0.040 \mathrm{~nm} / \mathrm{s}$, respectively. The measurements corresponding to the lowest rate $(0.013 \mathrm{~nm} / \mathrm{s})$ have been reported elsewhere. ${ }^{41}$ wafers, it has been found that a volume contribution is predominant below a critical thickness; Liedke et al. ${ }^{12}$ have studied the crossover from thin to thick film regimes and determined a critical thickness of $7 \mathrm{~nm}$ for cobalt layers. In the thick film regime, the role of the volume contribution is not significant; in fact, they have quantified a merely $\sim 1 \%$ of the surface contribution. To clarify the importance of the volume UMA in the present analysis, we performed in-plane angle dependent SQUID and longitudinal MOKE measurements of our as-grown films, which neither did reveal any in-plane anisotropy nor did other series of eroded films with a shallow ripple pattern (amplitude $\sim 1 \mathrm{~nm}$ ). Therefore, a volume contribution can be ignored hereafter.

Several magnetic effects other than dipolar interactions can be ignored in the analysis of the uniaxial anisotropy. As stated before, the fine grain polycrystal microstructure yields the magnetocrystalline anisotropy energy averaged out. Likewise, the residual film thickness is large as to neglect the relevance of a magnetization ripple state. ${ }^{40}$ Thus, our samples can be well described by a uniform magnetization throughout the film thickness. Schlömann calculated the in-plane demagnetizing field of such a magnetic film with an arbitrarily rough surface: on the assumption that the film surface is not too ragged, a periodic ripple profile with rms surface roughness $\omega_{\text {RMS }}$ and wavelength $\Lambda$ leads to the following formula for the uniaxial in-plane anisotropy field

$$
H_{S}=4 \pi \cdot M_{S} \cdot \frac{\pi \omega^{2} R M S}{\Lambda \cdot t} .
$$

We hereafter name the dimensionless quantity $\omega^{2} / \Lambda / t$ as Schlömann's parameter. The saturation field is proportional to Schlömann's parameter and the slope depends only on the saturation magnetization, $\mathrm{M}_{\mathrm{S}}$, of hcp cobalt. A minor reduction of $\mathrm{M}_{\mathrm{S}}$ could arise from magnetic frustration at grain boundaries, related either to the type of growth one expects (Volmer-Weber) or to the small effect of cobalt silicide formation or both. The set of four Hs data for continuous ripple films plotted in Figure 6 as a function of Schlömann's parameter can be fitted to a linear law (dashed line). This fit includes a small y-intercept that is null, within uncertainty $(0.04 \pm 0.09$ $\mathrm{kG})$. In accord with the measurements referred to above in different samples, there is no evidence of a volume contribution to UMA in the ripple films either. More importantly, from the slope of the linear regression, we obtain the saturation magnetization $\left(4 \pi \mathrm{M}_{\mathrm{S}}=1400 \pm 200 \mathrm{emu} / \mathrm{cm}^{3}\right)$, which compares well with the literature values for hcp cobalt. It gives a strong support to the surface-only origin for the UMA in the thick polycrystalline films. In the hard-axis configuration, the anisotropy field $H_{S}$ can be written as $H_{S}=2 \cdot K_{U} / M_{S}$, where $K_{U}$ is the UMA energy density. From this formula, we estimate $K_{U}$ as high as $\sim 5 \times 10^{5} \mathrm{ergs} / \mathrm{cm}^{3}$ for the roughest continuous films, which comparably exceeds the values of UMA energy density reported by other authors. Further, it could reach even higher values if the patterning process would apply a higher ion flux onto a thicker as-grown film.

Any dipolar contribution from the film-substrate interface can safely be ruled out because of its flat profile. X-ray reflectivity experiments of the virgin $100 \mathrm{~nm}$ cobalt films 


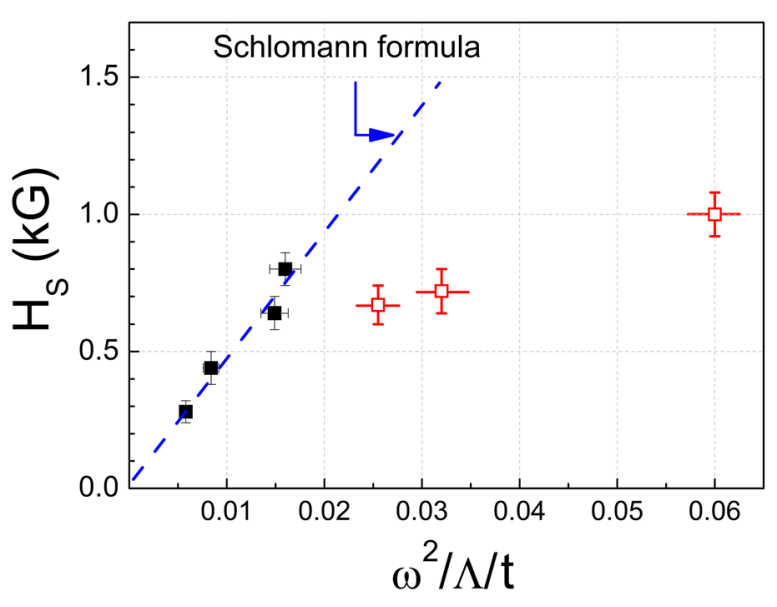

FIG. 6. Hard axis saturation field $\mathrm{Hs}$ as a function of the dimensionless parameter $\omega^{2} / \Lambda / t$ for continuous films (solid symbols) and discontinuous films (open symbols). The dashed straight line is a linear fit of the data for the continuous films in accord with the prediction for the demagnetizing field of a periodic ripple pattern (see text for details).

reported in a previous paper ${ }^{5}$ can be fitted with a structure model including a flat substrate $(\mathrm{rms}<0.2 \mathrm{~nm})$ and a rough top surface; interface alloying occurred to a probably minor extent without a significant roughening of the interface profile. Moreover, the use of films grown on different substrates such as glass and $\mathrm{MgO}\left(\begin{array}{lll}1 & 0 & 0\end{array}\right)$ led to anisotropy fields also in quantitative agreement with the Schlömann's prediction as that of films on Si ( $\left.\begin{array}{lll}1 & 0 & 0\end{array}\right)$-Figure 6. Thus, the film-substrate interface is not building surface magnetic charges in the continuous films.

Three open dots in Figure 6 are data for discontinuous ripple films. These Hs data for samples of very high ripple amplitude do not obey the expected linear law; rather, they remain at about $0.80-1.0 \mathrm{kG}$. One could try to explain it as due to intrinsic effects of a very ragged surface; Schlömann estimated the second-order corrections to the demagnetizing field as $\omega^{4} / \Lambda^{3} / \mathrm{t}$, also higher order terms, to be significant only at surface angles over $\sim 45^{\circ}$ with respect to the mean surface. ${ }^{21}$ However, our films are not so ragged. Although the mean surface angle of our films goes up with erosion rate, nonetheless it always stays below $\sim 17^{\circ}$. Moreover, histograms of surface angle (not shown) do not reveal any surface parts with values above $30^{\circ}$ in the steepest zones. Then we think that the departure from prediction is related to the discontinuous morphology. Although those samples are only discontinuous by about $10 \%$, as estimated from the cutoff of the amplitude histogram at an amplitude equal to the mean film thickness, exchange interactions should break up whereby the ripples are detached and the magnetic energy balance changes drastically in these zones. Zhang et al. ${ }^{17}$ applied a direct ion erosion to iron films and reached the substrate to a surface fraction of $43 \%$; the detached ripples were regarded as spatially separated nanorods and still exhibited UMA. The study of cobalt planar nanowires made by ripple detachment will be the aim of a separate paper.

\section{CONCLUSIONS}

We have successfully tailored cobalt films as a model system in which the dipolar-only origin of UMA can be checked with a ripple patterned top surface. At a first fabrication stage, the film growth process made up a fine-grain polycrystalline hcp structure, although it has presumably delivered enough energy to drive silicide formation at the surface of the flat silicon substrates. XPS composition analysis has shown that silicide formation gradually occurs at the interface and leads overall to silicon-rich, non-magnetic cobalt silicide, $\mathrm{Co}_{1-\mathrm{X}}-\mathrm{Si}_{\mathrm{X}}$ with $\mathrm{X}>0.7$ (magnetically dead layer). From the composition analysis, the thickness of such silicide is uncertain. Other evidences suggest that the amount of cobalt diffused into the whole silicide is small as not to reduce significantly the film saturation magnetization and keep the interface flat. At a second fabrication stage, IBS techniques sculpt a ripple pattern of large amplitude on the film surface using the method of tuning up the roughness by means of increasing the ion beam flux. This method was previously used by Chason et al. ${ }^{23}$ with low ion densities and is hereby checked with high ion densities. The choice of ion beam parameters has allowed making ripple patterns of a nearly fixed wavelength and different amplitudes up to $20 \mathrm{~nm}$, whereas yielding a nearly fixed residual thickness $(35-50 \mathrm{~nm})$.

The top surface is the only magnetically relevant interface as the ion induced pattern drives both the coercivity and the uniaxial magnetic anisotropy with the easy axis in the direction of ripple crests. An increasing dependence of the Hc data on the ripple amplitude suggests that the magnetization reversal in the easy axis occurs by wall movement hampered on the ripple crests. A well-defined uniaxial anisotropy sets in, if the pattern dimensions and film thickness imply a sufficiently high magnetic anisotropy density. We have studied the origin of the UMA by considering the ripple amplitude as the leading variable; in this regard, the saturation field data in the hard-axis direction fit the formula by Schlömann ${ }^{21}$ for the demagnetizing field of a rough surface. Since any volume contribution can be ruled out, the present analysis confirms the surface-only origin of UMA for films of continuous morphology. Other films of slightly discontinuous morphology do not obey the prediction. The second order corrections to Schlömann formula, arising from a locally very steep surface, do not explain the departure from prediction, which is believed to arise from magnetic energy unbalance associated with the ripple detachment.

\section{ACKNOWLEDGMENTS}

We strongly appreciate the assistance of Dr. J. Camarero to build our setup for vectorial MOKE magnetometry. This work has been funded on grants from Spanish institutions, Ministerio de Ciencia e Innovación (No. MAT2006-06242) and Junta de Comunidades de Castilla-La Mancha (No. PPII10-0054-1318). F.J.P. acknowledges funding through Grant Nos. MAT2010-18432 and CSD2008-00023.

\footnotetext{
${ }^{1}$ A. Keller and S. Facsko, Materials 3, 4811 (2010).

${ }^{2}$ M. A. Makeev, R. Cuerno, and A.-L. Barabasi, Nucl. Instrum. Methods Phys. Res., Sect. B 197, 185 (2002).

${ }^{3}$ A. Toma, D. Chiappe, B. Setina Batic, M. Godec, M. Jenko, and F. Buatier de Mongeot, Phys. Rev. B 78, 153406 (2008).

${ }^{4}$ P. Karmakar and D. Ghose, Surf. Sci. 554, L101 (2004).

${ }^{5}$ J. M. Colino and M. A. Arranz, Appl. Surf. Sci. 257, 4432 (2011).
} 
${ }^{6}$ K. Sato, I. Okamoto, Y. Kitamoto, and S. Ishida, Jpn. J. Appl. Phys., Part 1 46, 5139 (2007)

${ }^{7}$ J. Fassbender, T. Strache, M. O. Liedke, D. Markó, S. Wintz, K. Lenz, A. Keller, S. Facsko, I. Mönch, and J. McCord, New J. Phys. 11, 125002 (2009). ${ }^{8}$ M. Körner, K. Lenz, M. O. Liedke, T. Strache, A. Mücklich, A. Keller, S. Facsko, and J. Fassbender, Phys. Rev. B 80, 214401 (2009).

${ }^{9}$ M. O. Liedke, B. Liedke, A. Keller, B. Hillebrands, A. Mücklich, S. Facsko, and J. Fassbender, Phys. Rev. B 75, 220407(R) (2007).

${ }^{10}$ K. V. Sarathlal, D. Kumar, and A. Gupta, Appl. Phys. Lett. 98, 123111 (2011).

${ }^{11}$ E. Paz, F. Cebollada, F. J. Palomares, F. García-Sánchez, and J. M. González, Nanotechnology 21, 255301 (2010).

${ }^{12}$ M. O. Liedke et al., Phys. Rev. B 87, 024424 (2013).

${ }^{13}$ K. Chen, R. Frömter, S. Rössler, N. Mikuszeit, and H. P. Oepen, Phys. Rev. B 86, 064432 (2012).

${ }^{14}$ M. Körner, K. Lenz, R. A. Gallardo, M. Fritzsche, A. Mücklich, S. Facsko, J. Lindner, P. Landeros, and J. Fassbender, Phys. Rev. B 88, 054405 (2013).

${ }^{15}$ K. V. Sarathlal, D. Kumar, V. Ganesan, and A. Gupta, Appl. Surf. Sci. 258, 4116 (2012).

${ }^{16}$ K. Zhang, M. Uhrmacher, H. Hofsäss, and J. Krauser, J. Appl. Phys. 103, 083507 (2008).

${ }^{17}$ K. Zhang, F. Rotter, M. Uhrmacher, C. Ronning, J. Krauser, and H. Hofsäss, New J. Phys. 9, 29 (2007).

${ }^{18}$ M. T. Umlor, Appl. Phys. Lett. 87, 082505 (2005).

${ }^{19}$ N. L. Yakovlev, H. Chen, and K. Zhang, J. Nanosci. Nanotechnol. 11, 2575 (2011).

${ }^{20}$ A. F. Vaz, S. J. Steinmüller, and J. A. C. Bland, Phys. Rev. B 75, 132402 (2007).

${ }^{21}$ E. Schlömann, J. Appl. Phys. 41, 1617 (1970).

${ }^{22}$ M. A. Arranz and J. M. Colino, J. Phys.: Conf. Ser. 200, 072007 (2010).

${ }^{23}$ E. Chason, T. M. Mayer, and B. K. Kellerman, in Ion-solid interactions for materials modification and processing, edited by D. B. Poker, D. Ila, Y. T. Cheng, L. R. Harriott, and T.W. Sigmon (Mater. Res. Soc. Symp. Proc., 1996), Vol. 396, p. 143
${ }^{24}$ A. Redondo-Cubero, R. Gago, F. J. Palomares, A. Mücklich, M. Vinnichenko, and L. Vazquez, Phys. Rev. B 86, 085436 (2012).

${ }^{25}$ J. Camarero, J. Sort, A. Hoffmann, J. M. García-Martín, B. Dieny, R. Miranda, and J. Nogués, Phys. Rev. Lett. 95, 057204 (2005).

${ }^{26}$ J. M. Teixeira, R. Lusche, J. Ventura, R. Fermento, F. Carpinteiro, J. P. Araujo, J. B. Sousa, S. Cardoso, and P. P. Freitas, Rev. Sci. Instrum. 82, 043902 (2011).

${ }^{27}$ W. L. Chan and E. Chason, J. Appl. Phys. 101, 121301 (2007).

${ }^{28}$ J. M. Fallon, C. A. Faunce, P. J. Grundy, and H. J. Blythe, J. Appl. Phys. 87, 6833 (2000).

${ }^{29}$ T. Ishiguro, H. Fujii, Y. Ichinose, J. Endo, and H. Harada, J. Appl. Phys. 61(8), 4284 (1987).

${ }^{30}$ J. M. Fallon, C. A. Faunce, and P. J. Grundy, J. Phys.: Condens. Matter 12, 4075 (2000).

${ }^{31}$ M. Vélez, S. M. Valvidares, J. Díaz, R. Morales, and J. M. Alameda, IEEE Trans. Magn. 38(5), 3078 (2002).

${ }^{32}$ J. Díaz, R. Morales, S. M. Valvidares, and J. M. Alameda, Phys. Rev. B 72, 144413 (2005).

${ }^{33}$ P. L. Tam, Y. Cao, and L. Nyborg, Surf. Sci. 606, 329 (2012).

${ }^{34}$ M. Garcia-Mendez, F. F. Castillon, G. A. Hirata, M. H. Farias, and G. Beamson, Appl. Surf. Sci. 161, 61 (2000).

${ }^{35}$ V. Kinsinger, I. Dezsi, P. Steiner, and G. Langouche, J. Phys.: Condens. Matter 2, 4955 (1990)

${ }^{36}$ Y.-P. Zhao, R. M. Gamache, G.-C. Wang, and T.-M. Lu, J. Appl. Phys. 89, 1325 (2001).

${ }^{37}$ M. Li, G.-C. Wang, and H.-G. Ming, J. Appl. Phys. 83(10), 5313 (1998).

${ }^{38}$ J. Swerts, S. Vandezande, K. Temst, and C. Van Haesendonck, Solid State Commun. 131, 359 (2004).

${ }^{39}$ G. Palasantzas, Y.-P. Zhao, J. Th. M. De Hosson, and G.-C. Wang, Physica B 283, 199 (2000).

${ }^{40}$ K. D. Leaver, Thin Solid Films 2, 149-E (1968).

${ }^{41}$ M. A. Arranz, J. M. Colino, E. Jiménez, and J. Camarero, "Evidences of uniaxial magnetic anisotropy in nanopatterned cobalt films," in Proceedings of the International Conference on Nanoscale Pattern Formation at Surfaces, El Escorial, Spain,18-22 September, 2011. 